\title{
ESTIMATORS FOR INET-FORMATTED SOQPSK-TG
}

\author{
Michael Rice \\ Brigham Young University \\ Mohammad Saquib \\ University of Texas at Dallas \\ Erik Perrins \\ University of Kansas
}

\begin{abstract}
This paper presents algorithms for estimating the frequency offset, multipath channel coefficients, and noise variance of iNET-formatted SOQPSK-TG. The estimators compare the received signal samples corresponding to the iNET preamble and attached sync marker (ASM) bits to a locally stored copy of the SOQPSK-TG samples corresponding to the same. The mean and variance of the three estimators over ten test channels derived from channel sounding experiments at Edwards AFB is presented. The results show that usable estimates are achievable.
\end{abstract}

\section{INTRODUCTION}

Modern digital communication techniques leverage the capabilities of advanced digital signal processing capabilities to improve the BER performance of modulated carriers in harsh radio frequency (RF) environments. A fundamental element of these improvements is knowledge of the RF environment (e.g., frequency offset, multipath channel coefficients, noise variance). If the transmitted signal contains one or more known bit fields, then a sampled data receiver may compare the received signal corresponding to the known bits with a locally stored copy of the known bits to estimate the desired channel conditions. iNET-formatted SOQPSK-TG is an example of a transmitted signal containing a known bit pattern (in the form of preamble and ASM bits: see the top portion of Figure 1). This paper describes estimators for the frequency offset, multipath channel coefficients, and noise variance.

Approved for public release; distribution is unlimited. 412TW-PA-14245 
In what follows, boldface lowercase variables denote column vectors whereas boldface uppercase variables denote matrices. For the matrix $\mathbf{M}, \mathbf{M}^{\top}$ denotes the transpose of $\mathbf{M}$ where as $\mathbf{M}^{\dagger}$ denotes the Hermitian (conjugate-transpose) of $\mathbf{M}$.

The transmitted signal, $s(t)$, is SOQPSK-TG whose input bit stream is summarized in the top portion of Figure 1. The signal propagates through a frequency selective channel and experiences a frequency offset and the addition of additive white Gaussian noise. We assume the received signal is filtered, down-converted to complex baseband, and sampled (not necessarily in that order) using standard techniques. The resulting sequence of received samples is

$$
r(n)=\left[\sum_{k=-N_{1}}^{N_{2}} h(k) s(n-k)\right] e^{j \omega_{0} n}+w(n)
$$

where $h(n)$ is the impulse response of the unknown channel impulse response with support on $-N_{1} \leq n \leq N_{2}, \omega_{0}$ rads/sample is the frequency offset, and $w(n)$ is a proper [1] complex-valued zero-mean Gaussian random process with auto-covariance function

$$
\frac{1}{2} \mathrm{E}\left\{w(n) w^{*}(n-k)\right\}=\sigma_{w}^{2} \delta(k) .
$$

As an example of the need for these estimators, data-aided MMSE equalization requires estimates of $h(n)$ for $-N_{1} \leq n \leq N_{2}$ and $\sigma_{w}^{2}$ [2]. But good estimates for $h(n)$ and $\sigma_{w}^{2}$ require the received data to be de-rotated by an estimate of the frequency offset $\omega_{0}$ rads/sample. (A good estimate for $\omega_{0}$ is required for coherent detection as well.) The estimator described in this paper is a three step estimator:

1. A frequency offset estimate $\hat{\omega}_{0}$ is computed from the received samples $r(n)$ corresponding to the iNET preamble bits.

2. The channel coefficient estimates $\hat{h}(n)$ for $-N_{1} \leq n \leq N_{2}$ are computed using $\hat{\omega}_{0}$ and the received samples $r(n)$ corresponding to the iNET preamble and ASM bits.

3. The noise variance estimate is computed from $\hat{\omega}_{0}, \hat{h}(n)$, and the received samples $r(n)$ corresponding to the iNET preamble and ASM bits.

Each of these estimators are described in the following sections.

\section{FREQUENCY OFFSET ESTIMATOR}

Let $i$ be the index in $r(n)$ corresponding to the beginning of the preamble sequence and let $L_{p}$ be the number of samples in the preamble sequence. The situation is illustrated in Figure 1. The complicating factor here is the unknown channel impulse response $h(n)$. The difficulty of the presence of $h(n)$ may be overcome by exploiting the periodic properties of the iNET preamble.

Because the iNet preamble comprises 8 repetitions of a 16 bit sequence, the resulting SOQPSKTG modulated carrier is also periodic over the time interval corresponding to the occurrence of the 


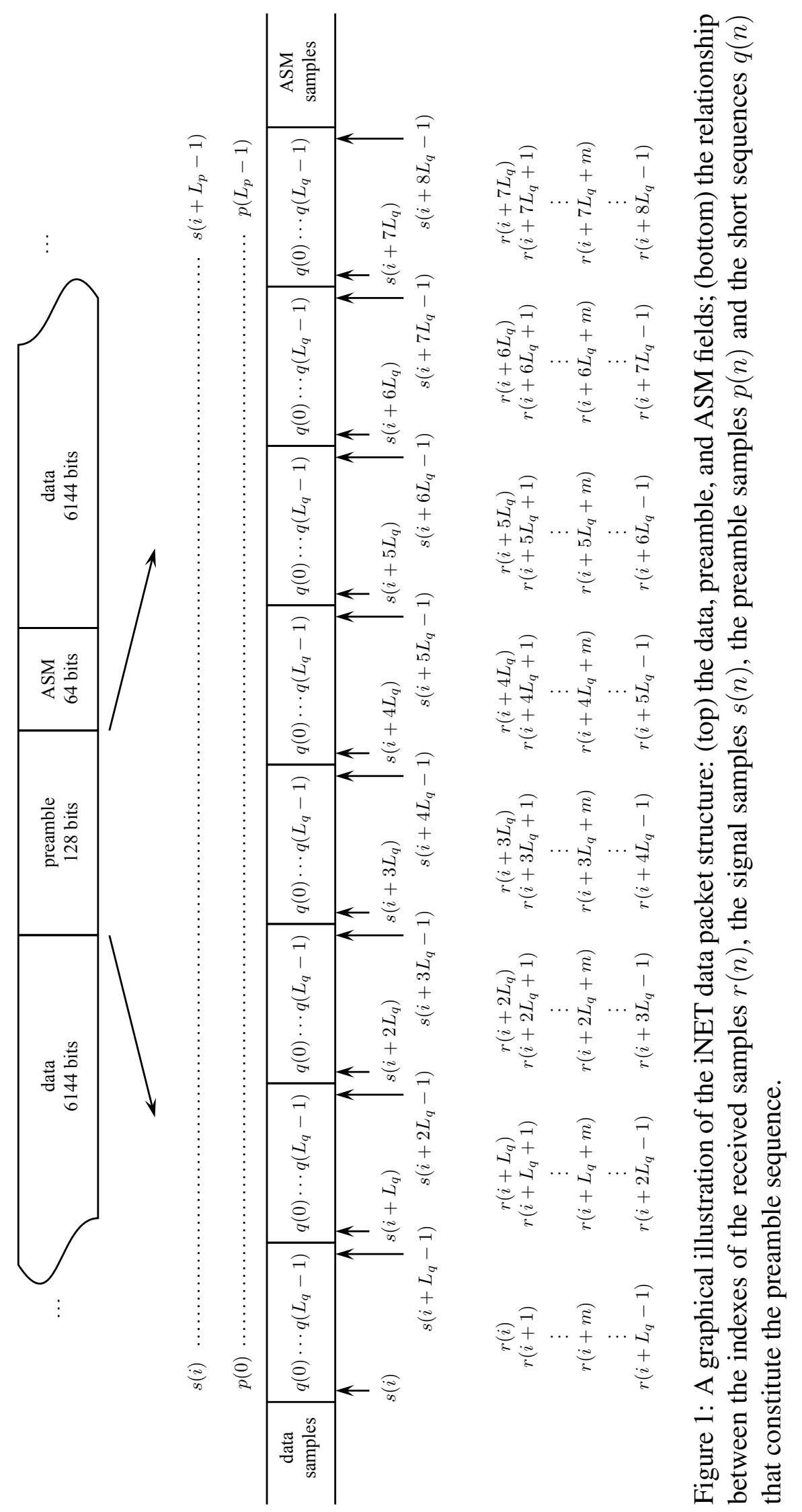


preamble. Furthermore the sampled SOQPSK-TG signal is periodic over the interval corresponding to the preamble sequence. The situation is illustrated in Figure 1. Here, $s(n)$ are the samples of the complex baseband SOQPSK-TG signal and $p\left(n^{\prime}\right)$ for $n^{\prime}=0,1, \ldots, L_{p}-1$ are the samples of the complex baseband SOQPSK-TG signal corresponding to the preamble bits. If the preamble sequence starts at index $i$, then $s(i)=p(0), s(i+1)=p(1)$ and so on to $s\left(i+L_{p}-1\right)=p\left(L_{p}-1\right)$. Now let $q(n)$ be the samples of the complex baseband SOQPSK-TG signal corresponding to the 16-bit sequence that is repeated to form the preamble, and let the length of $q(n)$ be $L_{q}$. (At an equivalent sample rate of 2 samples/bit, $L_{p}=256$ and $L_{q}=32$.)

Using a simple change of index, we may re-write $r(i), r(i+1), \ldots, r(i+L p-1)$ as $r\left(i+\ell L_{q}+m\right)$ for $\ell=0, \ldots, 7$ and $m=0, \ldots, L_{q}-1$. The contribution of $h(n)$ to $r\left(i+\ell L_{q}+m\right)$ depends only on $m$, the position inside the $q(\cdot)$ block. Consequently, the only difference (other than the additive noise) between the sample at position $m$ in adjacent blocks is the phase rotation due to the frequency offset. This feature is captured by the correlation function.

Assuming $N_{1}<L_{q}$ and $N_{2}<L_{q}$, we are interested in the correlation function involving the middle six blocks:

$$
R(\delta)=\frac{1}{6 L_{q}-\delta} \sum_{n=i+L_{q}+\delta}^{i+7 L_{q}-1} r(n) r^{*}(n-\delta)
$$

Because the samples $r(n)$ are periodic with period $L_{q}$, we are interested in evaluating this function at integer multiples of $L_{q}$. For $\delta=d L_{q}(1 \leq d \leq 5)$ the correlation is

$$
R\left(d L_{q}\right)=\frac{\alpha^{2}}{L_{q}} e^{j d L_{q} \omega_{0}}+v
$$

where $\alpha^{2}$ is given by

$$
\alpha^{2}=\sum_{m=0}^{L_{q}-1}|\alpha(m)|^{2}
$$

and $v$ is approximately a zero-mean complex-valued Gaussian random variable. Inspired by the delay-and-multiply (D\&M) estimator described by Eq. (4.5.1) of [3], we use a frequency estimator based on (4) corresponding to $d=1$ :

$$
\hat{\omega}_{0}=\frac{1}{L_{q}} \arg \left\{R\left(L_{q}\right)\right\} .
$$

This estimator is simple and exploits the noise averaging that occurs over the $5 L_{q}$ consecutive samples of $r(n)$. 


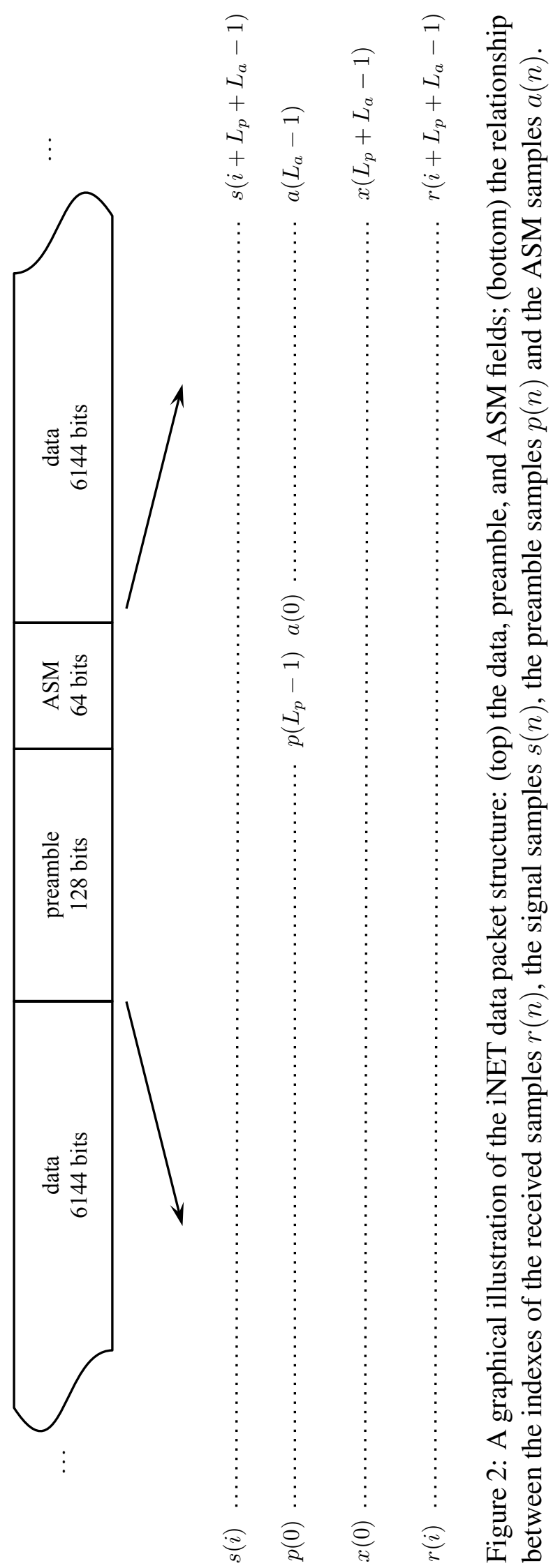




\section{CHANNEL ESTIMATOR}

The channel estimator is based on the SOQPSK-TG samples corresponding to the iNET preamble and ASM fields. The situation illustrated in Figure 2. Because the channel estimator does not exploit the periodic nature of the preamble, the periodic elements of the preamble sequence are not made explicit here. For notational purposes, we let $x(0), \ldots, x\left(L_{p}+L_{a}-1\right)$ represent the samples corresponding to the preamble followed by the ASM bits.

The first step is to remove the frequency offset from the sequence of received samples. This is accomplished using

$$
r_{d}(n)=r(n) e^{-j \hat{\omega}_{0} n}, \quad n=i, \ldots, i+L_{p}+L_{a}+L_{d}-1
$$

where $L_{d}=N \times 6144$ is the number of samples corresponding to the 6144 data bits. (Here the subscript $d$ means "de-rotation.")

The next step is to organize the $r_{d}(n)$ corresponding to the preamble and ASM bits in to a vector, but skipping the first $N_{2}$ samples and last $N_{1}$ samples:

$$
\mathbf{r}_{d}=\left[\begin{array}{c}
r_{d}\left(i+N_{2}\right) \\
\vdots \\
r_{d}\left(i+L_{p}+L_{a}-N_{1}-1\right)
\end{array}\right] .
$$

This vector is related to the samples corresponding to the preamble and ASM samples by

$$
\mathbf{r}_{d}=\mathbf{X h}+\mathbf{w}
$$

where $\mathbf{X}$ be the $\left(N_{p}+N_{a}-N_{1}-N_{2}\right) \times\left(N_{1}+N_{2}+1\right)$ convolution matrix formed from these samples:

$$
\mathbf{X}=\left[\begin{array}{ccc}
x\left(N_{2}+N_{1}\right) & \cdots & x(0) \\
\vdots & & \vdots \\
x\left(L_{p}+L_{a}-1\right) & \cdots & x\left(L_{p}+L_{a}-N_{1}-N_{2}-1\right)
\end{array}\right]
$$

and where $\mathbf{h}$ is the $\left(N_{1}+N_{2}+1\right) \times 1$ vector formed by the channel coefficients,

$$
\mathbf{h}=\left[\begin{array}{lll}
h\left(-N_{1}\right) & \cdots & h\left(N_{2}\right)
\end{array}\right]^{\top},
$$

and $\mathbf{w}$ is the $\left(L_{p}+L_{a}-N_{1}-N_{2}\right) \times 1$ vector of noise samples. From this, the vector of channel estimates is

$$
\hat{\mathbf{h}}=\left(\mathbf{X}^{\dagger} \mathbf{X}\right)^{-1} \mathbf{X}^{\dagger} \mathbf{r}_{d}
$$

This is only true for middle six blocks (i.e., $\ell=1, \ldots, 6)$ because the convolution sum in the first block $(\ell=0)$ includes samples from the data field immediately preceding the first block whereas the last block $(\ell=7)$ includes samples from the ASM field immediately following the last block. Eliminating the first and last blocks is sufficient as long as $N_{1}<L_{q}$ and $N_{2}<L_{q}$. If either of these conditions are not true, then this is true for fewer blocks.

Because the preamble is an 8-times repetition of $\mathrm{CD} 98_{\text {hex }}$, the convolution matrix formed from the samples corresponding to the preamble is not sufficiently well-conditioned to provide robust channel estimates in the presence of even a small frequency offset. The convolution matrix formed from samples corresponding to both the preamble and ASM fields has less structure and provides more robust channel estimates. See Section 4 of [4]. 
At first glance, the computational complexity of (12) seems quite high. But note that the matrix $\mathbf{X}$ is fixed. Consequently, the matrix product $\left(\mathbf{X}^{\dagger} \mathbf{X}\right)^{-1} \mathbf{X}^{\dagger}$ may be precomputed and stored at the receiver. The result is that the channel estimator reduces to a matrix-vector multiplication.

\section{NOISE VARIANCE ESTIMATOR}

The noise variance may be computed from a scaled version of the sample variance corresponding to the error between the observed signal and its estimate. In matrix/vector form, the sample variance is the inner product of a vector, divided by its length. The noise variance estimator is

$$
\hat{\sigma}_{w}^{2}=\frac{1}{2 \rho}\left|\mathbf{r}_{d}-\mathbf{X} \hat{\mathbf{h}}\right|^{2}
$$

where $\mathbf{r}_{d}$ is given by (9), $\mathbf{X}$ by (10), and $\hat{\mathbf{h}}$ by (12). The scale factor $\rho$ is

$$
\rho=\operatorname{Tr}\left\{\mathbf{I}-\mathbf{X}\left(\mathbf{X}^{\dagger} \mathbf{X}\right)^{-1} \mathbf{X}^{\dagger}\right\}
$$

where $\mathbf{I}$ is the $\left(N_{p}+N_{a}-N_{1}-N_{2}\right) \times\left(N_{p}+N_{a}-N_{1}-N_{2}\right)$ identity matrix. This scale factor is chosen to produce an unbiased estimate of the noise variance (see Section 7 of [4]).

\section{PERFORMANCE}

The performance of the three estimators was evaluated in compute simulation using the following parameters:

1. The payload data rate was equivalent to $10 \mathrm{Mbits} / \mathrm{s}$ (the equivalent "over-the-air" bit rate was 10.3125 Mbits/s). The iNET-formatted SOQPSK-TG signal and channel were generated at an equivalent sample rate of 2 samples/bit.

2. Because the channel estimator does not know the true length of the channel, the estimator used values for $N_{1}$ and $N_{2}$ larger than any of the test channels. These values were $N_{1}=12$ and $N_{2}=25$ samples.

3. The simulations were performed over 10 representative channels derived from channel sounding measurements conducted at Edwards AFB under the M4A program [5]. The test channels are summarized in Table 1 and the corresponding frequency-domain plots are shown in Figure 3.

The results for the frequency offset estimator (6) are shown in Figures 4 and 5. The simulated estimator means for frequency offsets $-50 \mathrm{kHz} \leq \Delta f \leq 50 \mathrm{kHz}$ over the ten test channels are plotted in Figure 4. This figure shows that the frequency estimator is unbiased and capable of accurately estimating the frequency offset over this range. The simulated variance of the frequency offset estimator (6) is shown in Figure 5 where it is observed that the estimator error variance decreases with increasing $E_{b} / N_{0}$, as expected. The RMS frequency error is the square root of the 
Table 1: Description of the ten test channels used in the simulations.

\begin{tabular}{crrrl}
\hline channel & $N_{1}$ & $N_{2}$ & length & environment \\
\hline 01 & 1 & 7 & 9 & Taxiway E \\
02 & 2 & 17 & 20 & Taxiway E \\
03 & 1 & 22 & 24 & Taxiway E \\
04 & 5 & 13 & 19 & Takeoff on 22L \\
05 & 1 & 1 & 3 & Cords Road \\
06 & 1 & 2 & 4 & Cords Road \\
07 & 0 & 4 & 5 & Cords Road \\
08 & 2 & 3 & 6 & Black Mountain \\
09 & 1 & 1 & 3 & Black Mountain \\
10 & 2 & 3 & 6 & Land on 22L \\
\hline
\end{tabular}

variance. The plots show that for $E_{b} / N_{0} \geq 12 \mathrm{~dB}$, the RMS frequency error is a few hundred $\mathrm{Hz}$. This is small, but a mechanism for tracking out the residual frequency offset in the SOQPSK-TG detector is still required. (For example, see Figure 3 of [2]).

The results for the channel estimator (12) are shown in Figures 6 and 7. Here, the figure of merit is based on the sum of estimation errors:

$$
\mathcal{E}_{h}=\sum_{n=-N_{1}}^{N_{2}}[h(n)-\hat{h}(n)] .
$$

In Figure 6, the error mean is the expected value of $\mathcal{E}_{h}$ which, in turn, was estimated in simulation using the sample mean. In Figure 7, the error variance is the variance of $\mathcal{E}_{h}$ which, in turn, was estimated in simulation using the sample variance. The impact of the frequency offset estimation errors on the accuracy of the channel estimates is clearly evident. The estimator error mean only decreases with increasing $E_{b} / N_{0}$ when $E_{b} / N_{0} \geq 15 \mathrm{~dB}$.

The results for the noise variance estimator (13) are shown in Figures 8 and 9. Because the noise variance decreases with increasing $E_{b} / N_{0}$, we plot the normalized versions of the noise variance estimator error:

$$
\mathcal{E}_{w}=\frac{\sigma_{w}^{2}-\hat{\sigma}_{w}^{2}}{\sigma_{w}^{2}} .
$$

As with the channel estimator, the noise variance estimator clearly suffers from the frequency and channel estimation errors. Consequently, of the three estimates, this one is least reliable.

How these estimation errors impact the overall bit error rate (BER) is discussed in detail in [4]. The impact of the estimation errors may also be inferred from the (BER) performance of the MMSE equalizer presented in [2] where it is demonstrated that the equalization techniques enabled by these estimates have reasonably good BER performance over the same set of ten test channels. 


\section{CONCLUSIONS}

This paper presents algorithms for estimating the frequency offset (6), multipath channel coefficients (12), and noise variance (13) of iNET-formatted SOQPSK-TG. The estimators compare the received signal samples corresponding to the iNET preamble and attached sync marker (ASM) bits to a locally stored copy of the SOQPSK-TG samples corresponding to the same. The mean and variance of the three estimators over ten test channels derived from channel sounding experiments at Edwards AFB is presented. The impact of the estimation errors may be inferred from the (BER) performance of the MMSE equalizer presented in [2] where it is demonstrated that the equalization techniques enabled by these estimates have reasonably good BER performance over the same set of ten test channels.

\section{ACKNOWLEDGEMENTS}

This work was funded by the Test Resource Management Center (TRMC) Test and Evaluation Science and Technology (T\&E/S\&T) Program through the U.S. Army Program Executive Office for Simulation, Training and Instrumentation (PEO STRI) under contract W900KK-13-C-0026 (PAQ).

\section{REFERENCES}

[1] P. Schreier and L. Scharf, Statistical Signal Processing of Complex-Valued Data. New York: Cambridge University Press, 2010.

[2] M. Rice, M. S. Afran, M. Saquib, A. Cole-Rhodes, and F. Moazzami, "A comparison of three equalization techniques for iNET-formatted SOQPSK-TG," in Proceedings of the International Telemetering Conference, San Diego, CA, October 2014.

[3] U. Mengali and A. D'Andrea, Synchronization Techniques for Digital Receivers. New York: Springer, 1997.

[4] M. Rice, "Phase 1 report: Preamble assisted equalization for aeronautical telemetry (PAQ)," Brigham Young University, Tech. Rep., 2014, submitted to the Spectrum Efficient Technologies (SET) Office of the Science \& Technology, Test \& Evaluation (S\&T/T\&E) Program, Test Resource Management Center (TRMC). Also available on-line at http://hdl.lib.byu.edu/1877/3242.

[5] M. Rice and M. Jensen, "A comparison of L-band and C-band multipath propagation at Edwards AFB," in Proceedings of the International Telemetering Conference, Las Vegas, NV, October 2011. 

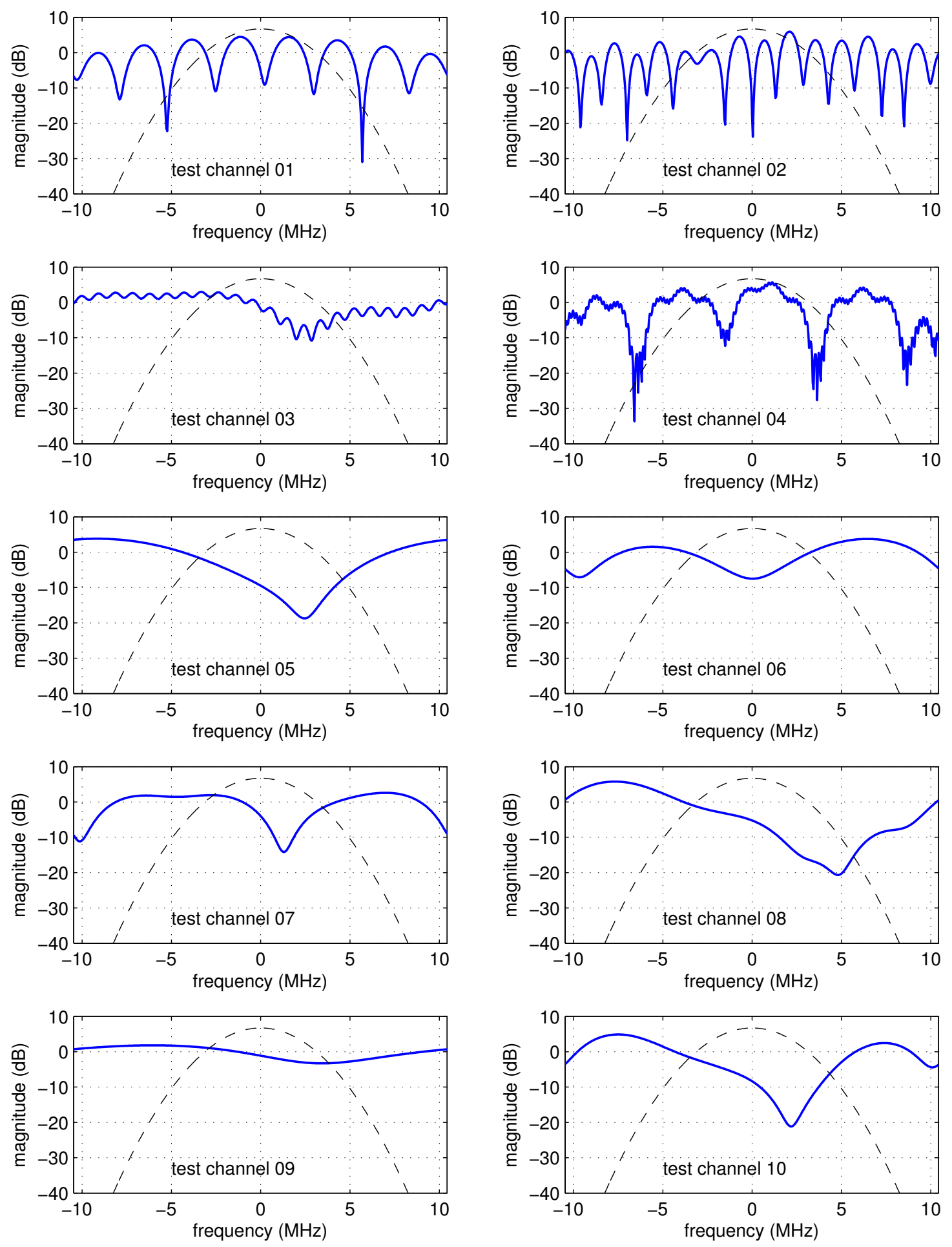

Figure 3: Frequency-domain plots of the example channels from channel sounding experiments at Edwards AFB. In each plot, the thick line is the channel frequency response and the thin line is the power spectral density of SOQPSK-TG operating at $10.3125 \mathrm{Mbits} / \mathrm{s}$. 

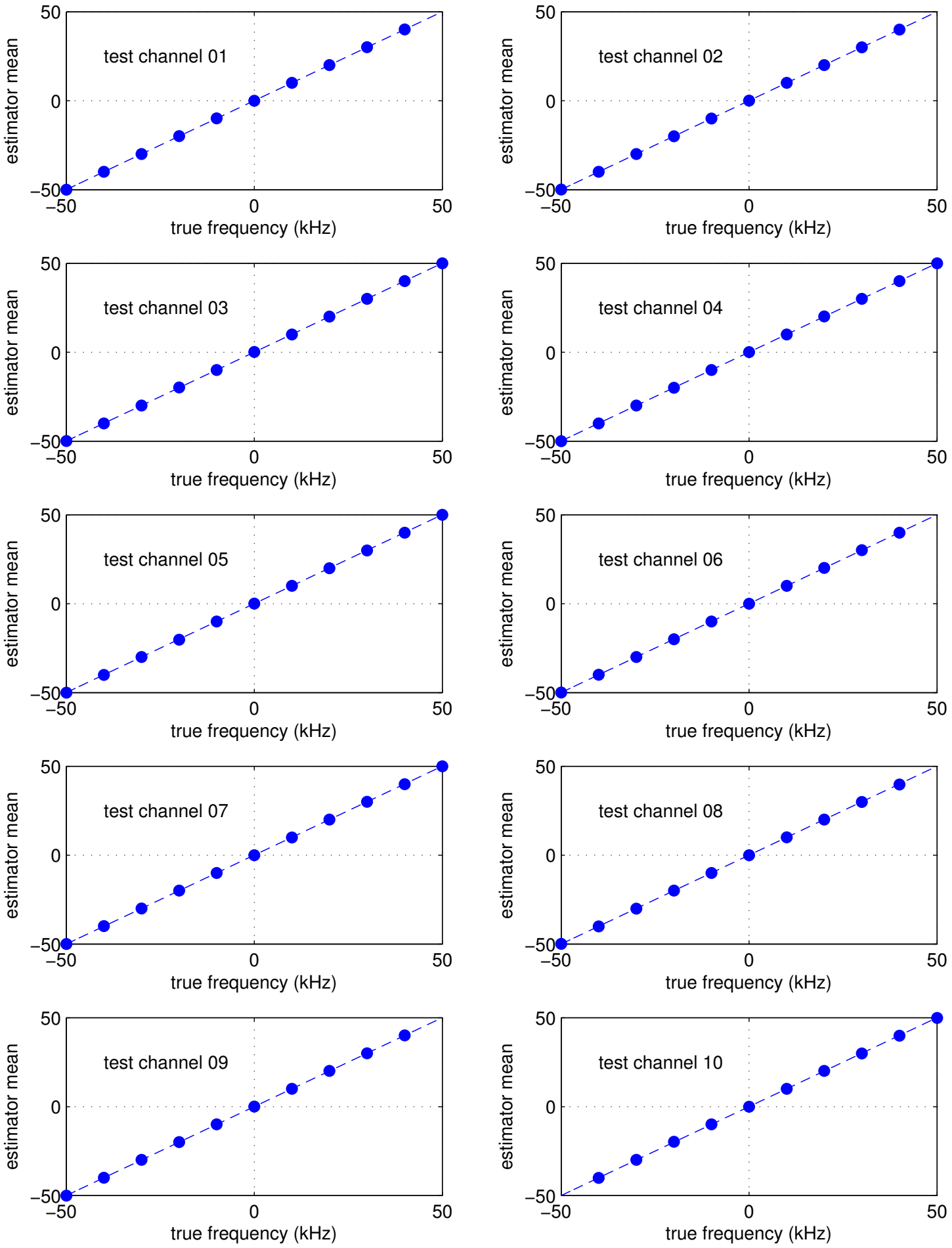

Figure 4: Estimator means for the frequency estimator (6) for the ten test channels described in Figure 3. 

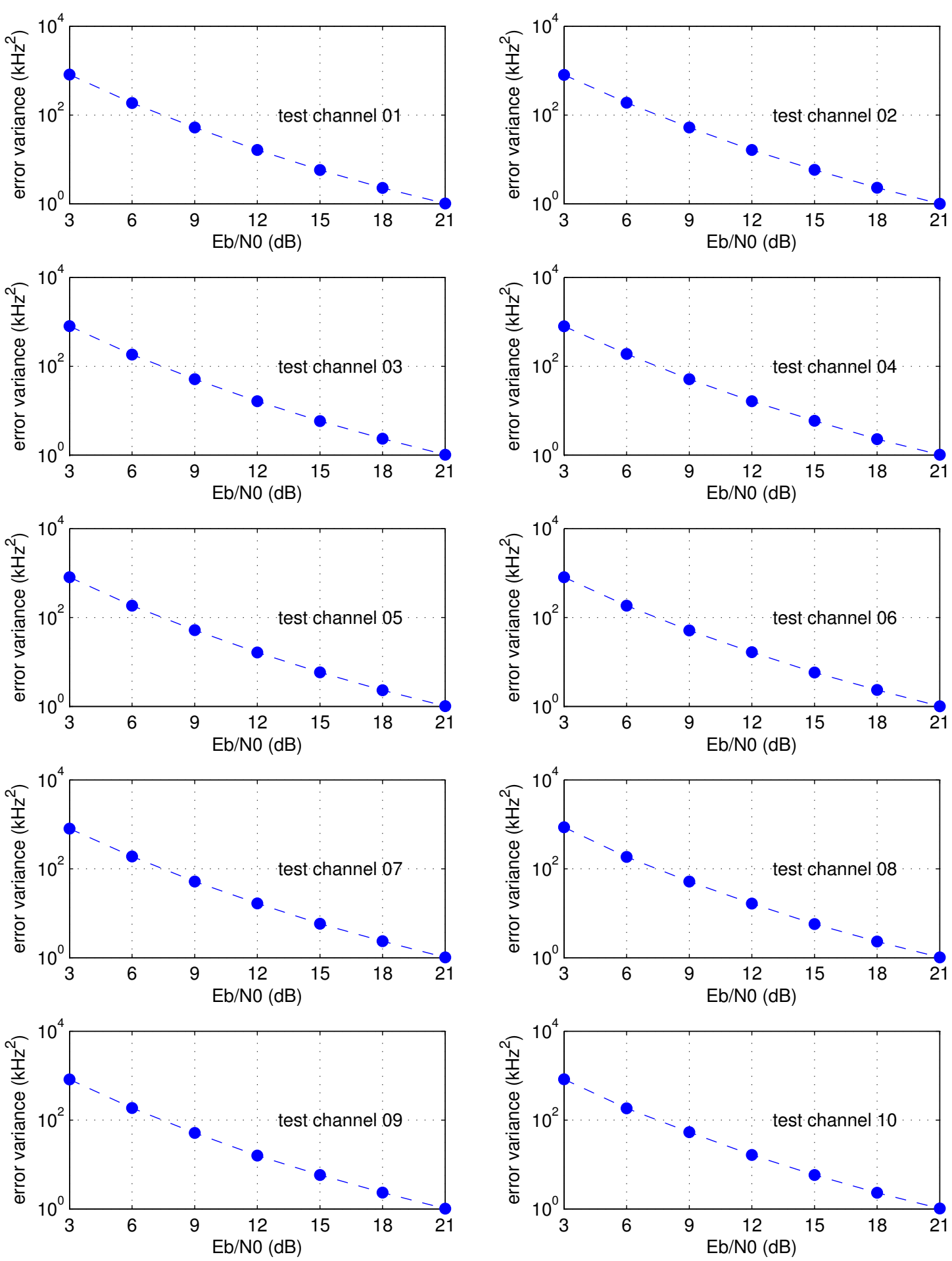

Figure 5: Estimator error variance for the frequency estimator (6) for the ten test channels described in Figure 3. 

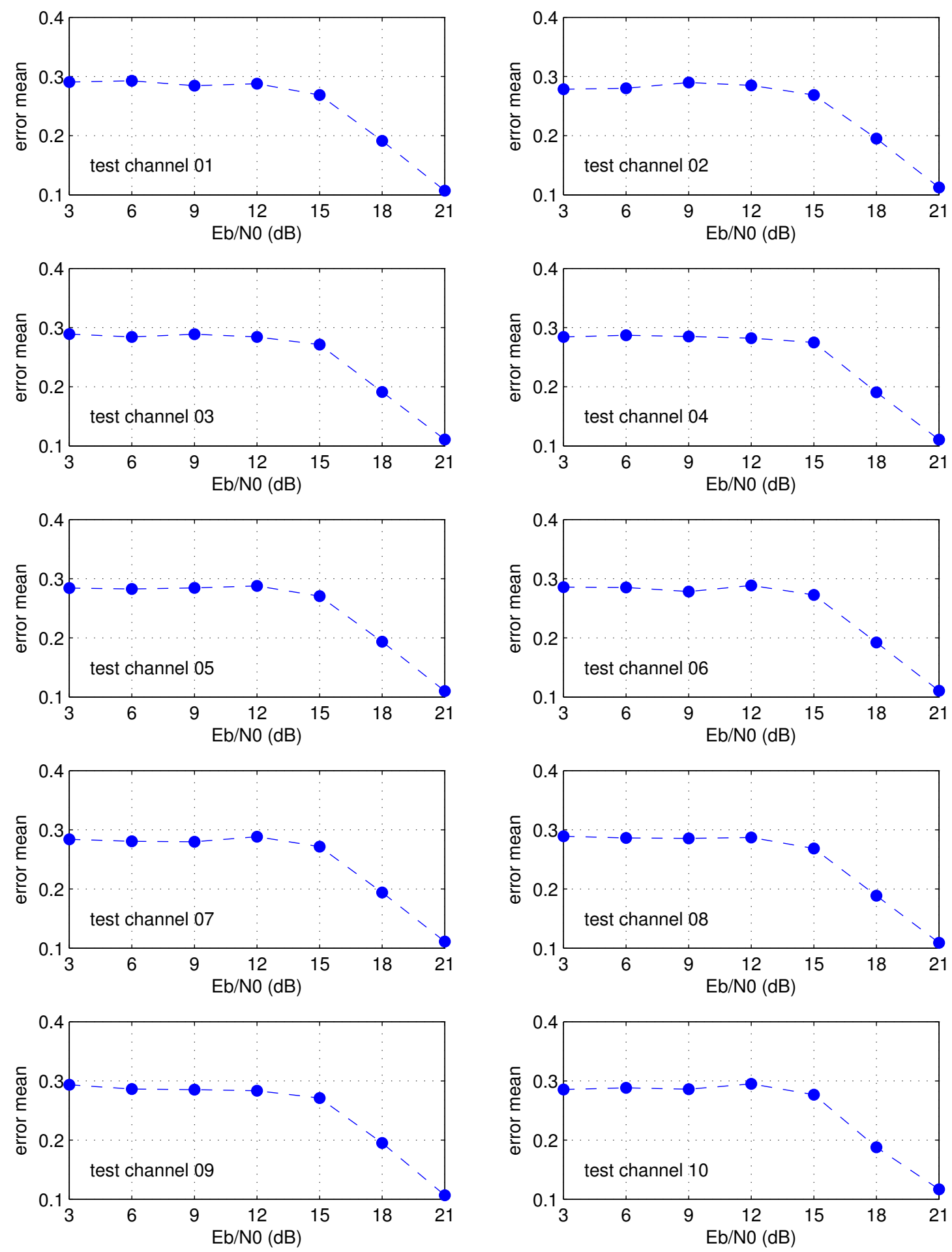

Figure 6: Estimator means for the channel estimator (12) for the ten test channels described in Figure 3. 

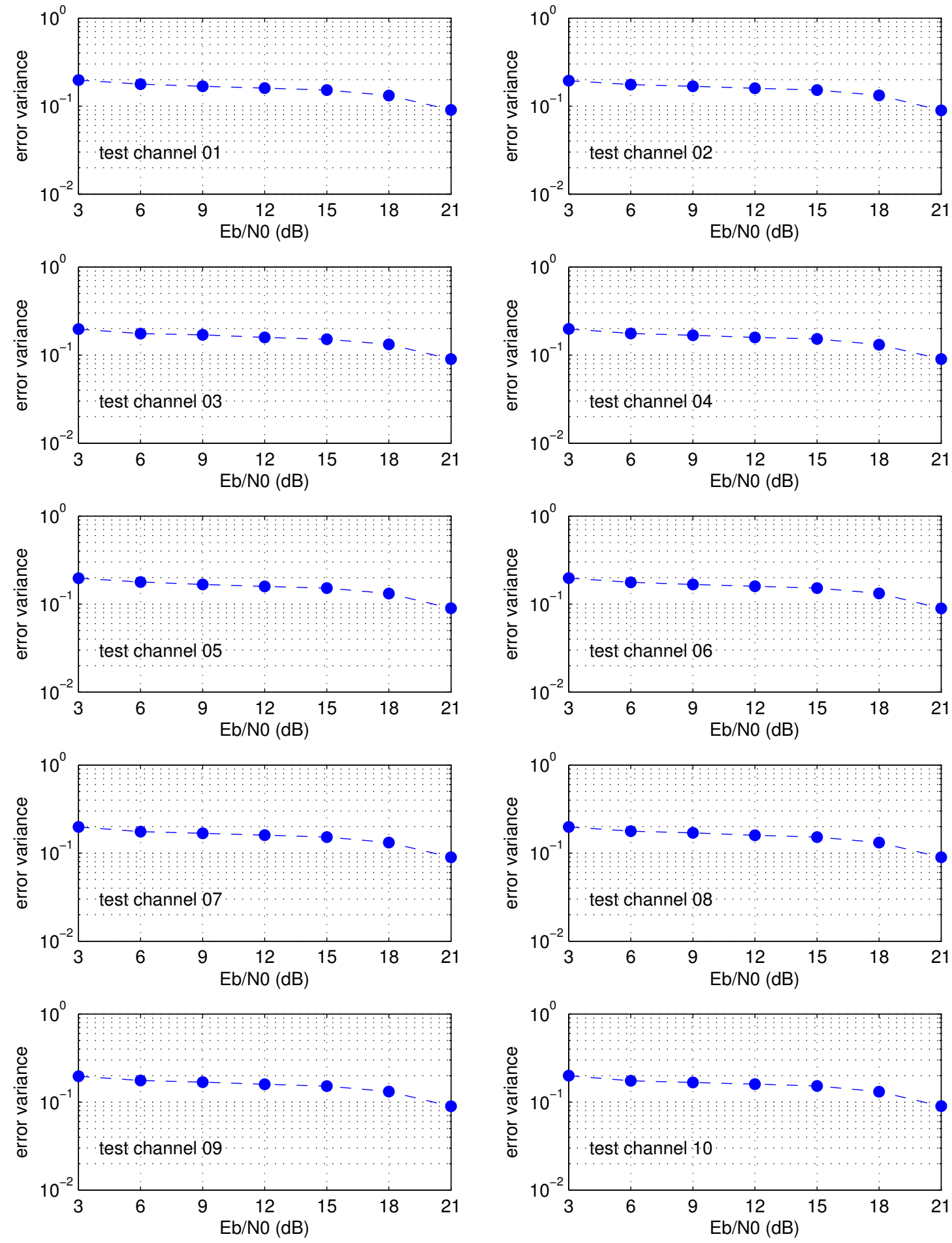

Figure 7: Estimator error variance for the channel estimator (12) for the ten test channels described in Figure 3. 

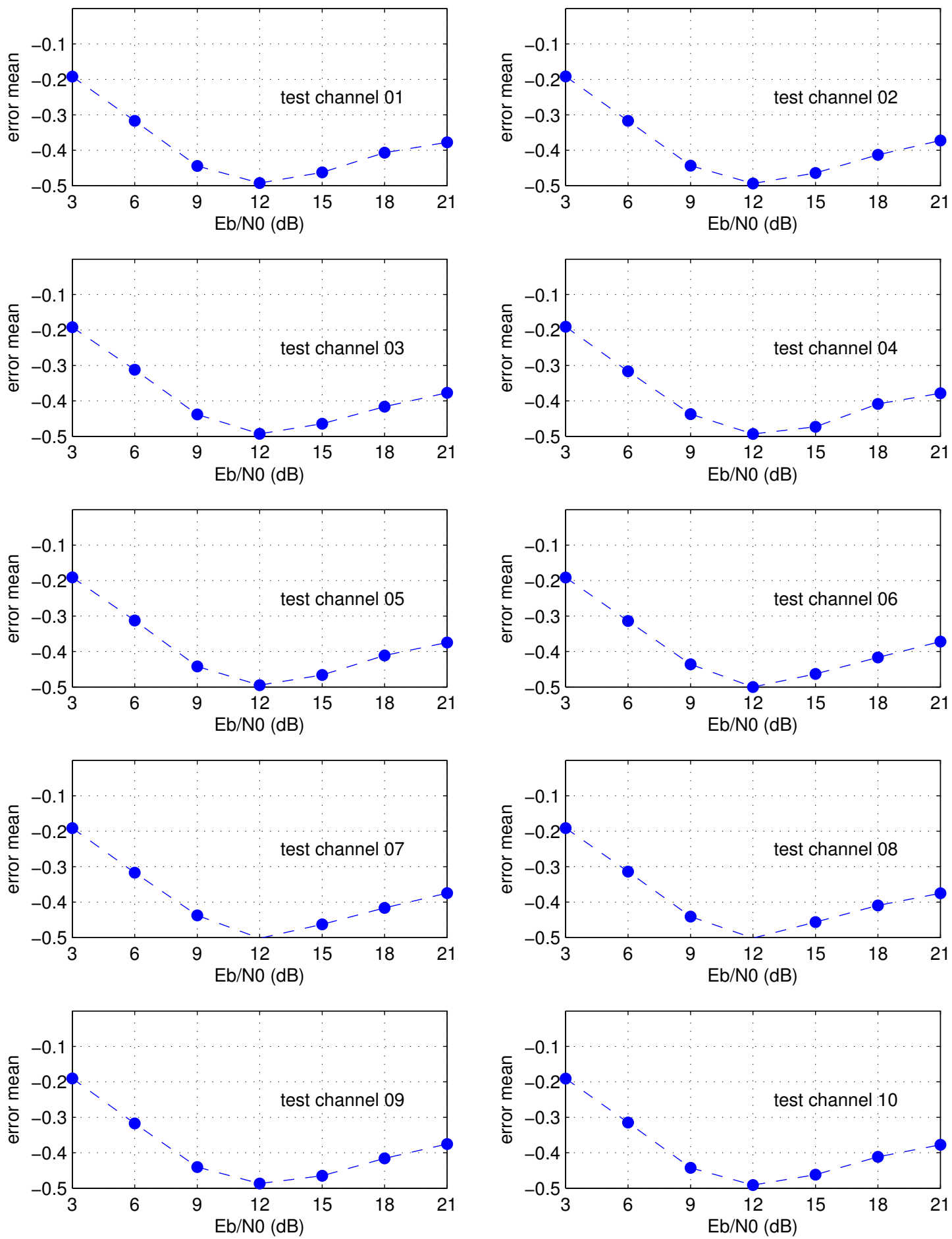

Figure 8: Estimator means for the noise variance estimator (13) for the ten test channels described in Figure 3. 

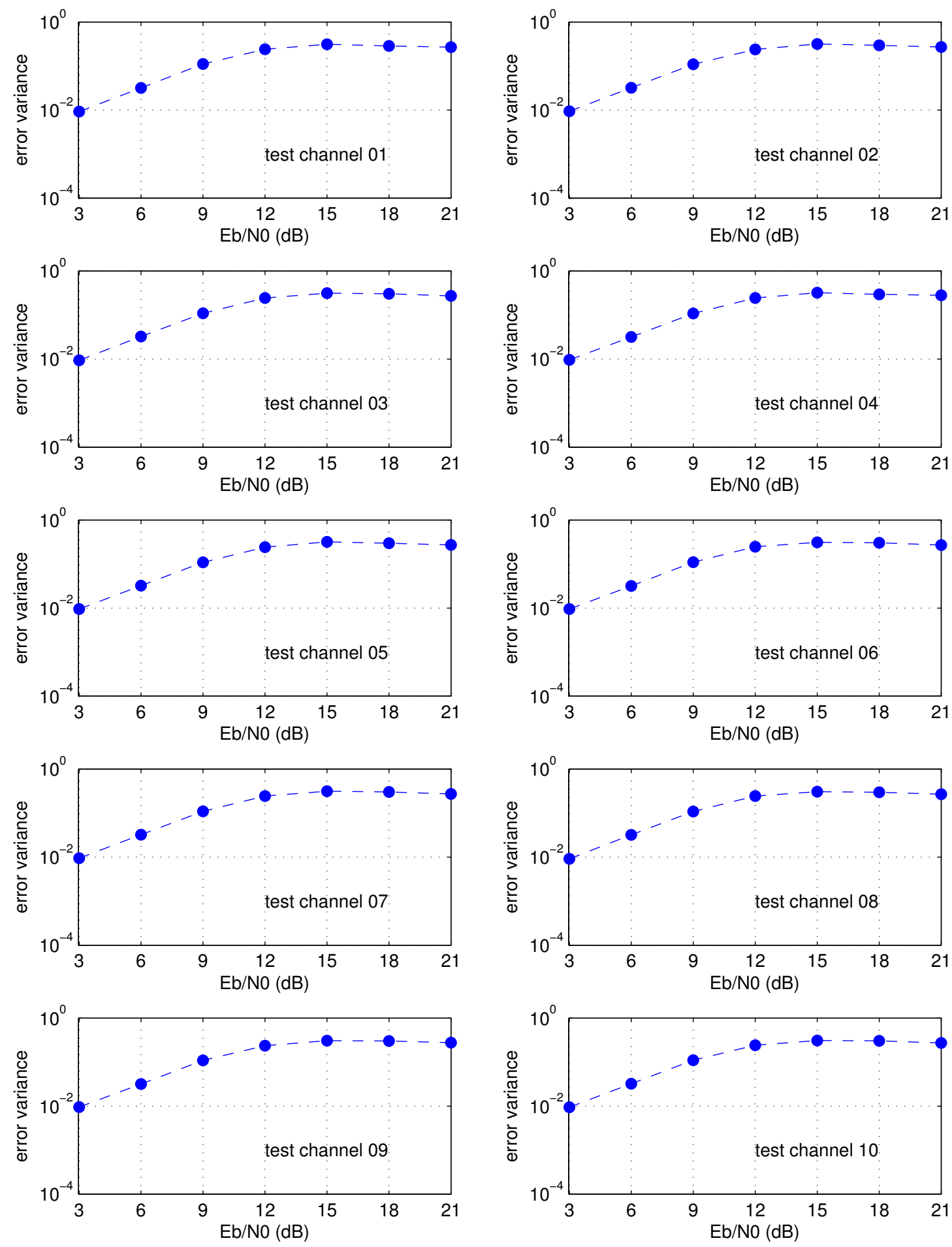

Figure 9: Estimator error variance for the noise variance estimator (13) for the ten test channels described in Figure 3. 